\title{
Evaluation of the motion of surgical instruments during intraocular surgery
}

J-P Hubschman ${ }^{1,2}$, J Son², B Allen², SD Schwartz ${ }^{1,2}$ and J-L Bourges ${ }^{1,2}$

\begin{abstract}
Purpose Robot assistance in ocular microsurgery could improve precision, dexterity, save time or prevent complications by task automation, and provide access to ocular surgery in undeserved countries by teleoperation. However, to design robotic devices, the range of motion of surgical instruments needs to be precisely quantified. Methods An electromagnetic tracking system was developed for intraocular surgery in order to quantify the movements of ophthalmic surgeons. Kinematics of surgical steps during phacoemulsification and pars plana vitrectomy procedures were determined by measuring the maximum translation and angular range of motion of intraocular surgical tools in the three planes.

Conclusion Important variations in amplitudes of rotation and translation were measured between both hands and between surgical tasks. These parameters may be used to develop a robotic intraocular surgical system or to improve training. Eye (2011) 25, 947-953; doi:10.1038/eye.2011.80; published online 29 April 2011
\end{abstract}

Keywords: sensor; electromagnetic sensor; ocular surgery; intraocular surgery; robot; surgical motion

\section{Introduction}

Robotic surgical systems have gained popularity over the past years in many surgical fields including urology, cardiology, gynecology, and digestive surgery. The da Vinci surgical robot, developed by Intuitive Surgical Inc. (Sunnyvale, CA, USA), is the main robotic surgical system commercialized today. Originally designed for laparoscopic surgery, this system has been tested in ophthalmology for extraocular and intraocular procedures. ${ }^{1-5}$ Ocular surgery could benefit from robotic systems, which, among other advantages, reduce tremor, enhance precision, and may integrate pre-operative imagery during surgery in real time. However, despite very promising potential, some limitations have hampered further use of surgical robots, mainly because the design of the remote center of motion is not suited for intraocular surgery. These observations led us to develop new surgical systems especially dedicated to eye surgery. These robotic platforms would either work solo or together with the da Vinci system. To define the specific characteristics of such systems, the first step is to quantify the range of motion of microsurgical tools during surgical procedures. To achieve this goal, we placed electromagnetic sensors on microsurgical instruments and recorded the instruments' motions during typical steps of anterior segment and posterior segment intraocular surgery.

\section{Materials and methods}

\section{Electromagnetic motion capture}

The electromagnetic motion capture (Figure 1) included magnetic sensors connected to a control unit and an active source magnet (microBird, Ascension, Burlington, VT, USA) (Figure 1a).

The three-axis magnetic sensors contained three orthogonally oriented coils encased in an epoxy-based coating, which formed a basic three-axis ring core fluxgate magnetometer (Figure 1b). Each sensor measured $1.3 \mathrm{~mm}$ in diameter and $5 \mathrm{~mm}$ in length. The active source electromagnetic unit consisted of three orthogonally oriented coils excited by a multimillisecond-pulsed DC current applied to a single coil at a time. The active electromagnet unit was rigidly attached to the surgical field next to the styrofoam human head. Sensors
'Department of Ophthalmology, Retina Division, Jules Stein Eye Institute, David Geffen School of Medicine at UCLA, Los Angeles, CA, USA

${ }^{2}$ Center for Advanced Surgical and Interventional Technology (CASIT), LOS Angeles, CA, USA

Correspondence: J-P Hubschman, Department of Ophthalmology, Retina Division, Jules Stein Eye Institute, UCLA, 100 Stein Plaza, Los Angeles, CA 90095-7000, USA Tel: + 1310206 5004; Fax: + 13107947905 E-mail: Hubschman@ jsei.ucla.edu

Received: 28 October 2010 Accepted in revised form: 15 February 2011 Published online: 29 April 2011

Meeting Presentation: This research was presented in part at the Association for Research in Vision and Ophthalmology, Annual Meeting, 2009. 
were optimally detected within a $0.5-\mathrm{m}$ radius from the electromagnetic unit, within the confines of the dry box. According to the manufacturer data sheet, for a working distance of $0.305 \mathrm{~m}$, the static resolution of the sensors was $0.5 \mathrm{~mm}$ for position and $0.1^{\circ}$ RMS angular for orientation. The output from the control unit provided 90 measurements of position and orientation per second.

Electromagnetic systems are sensitive when metallic objects are in the vicinity. Therefore, before carrying experimentations, the system was tested for reliability in order to obtain values, which served for further system calibration. Each sensor was translated between two points of known Cartesian coordinate in the three axes. The sensors were displaced $0.8-\mathrm{cm}$ and $19.8-\mathrm{cm}$ away

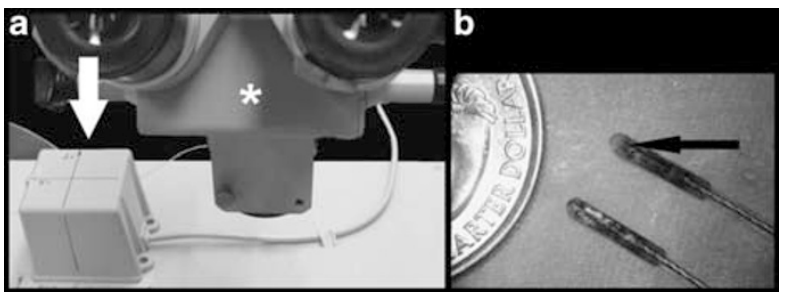

Figure 1 Porcine eyes were operated on under operating microscope (asterisk) at close vicinity of the electromagnetic source emitting a polarized magnetic field (a, white arrow) exiting small motion sensors (b, black arrow), which consisted of metallic coils encased in resin shell. from each other to test short translations and large translations, respectively. Each translation was performed 10 times to test repeatability. Each set was repeated by two operators (O1: JLB; O2: JPH) within two separated sessions (A and $\mathrm{B}$ ) to, respectively, test reproducibility across operators and reproducibility in time. On the basis of reliability test results, experiments were carried out on porcine eyes in a steady-state environment with regard to objects made of magnetizable metal (table, microscope, surgical instruments, surgical device console), and their proximity to sensors or the electromagnetic source.

\section{Surgical task design and tools}

For the purpose of the study, we defined five separated steps out of typical anterior (phacoemulsification) and posterior (vitrectomy) segment surgery (Figure 2).

Procedures were performed on freshly collected porcine eyes, affixed on Styrofoam human head. Selected phacoemulsification steps consisted of a lens sculpture (LS), based on divide and conquer nuclear-fracturing technique, an out-of-the bag lens removal (LR) and a cortex removal in infusion-aspiration (I/A) mode. Vitrectomy steps consisted of a $360^{\circ}$ peripheral pars plana vitrectomy (PPV) and aspiration

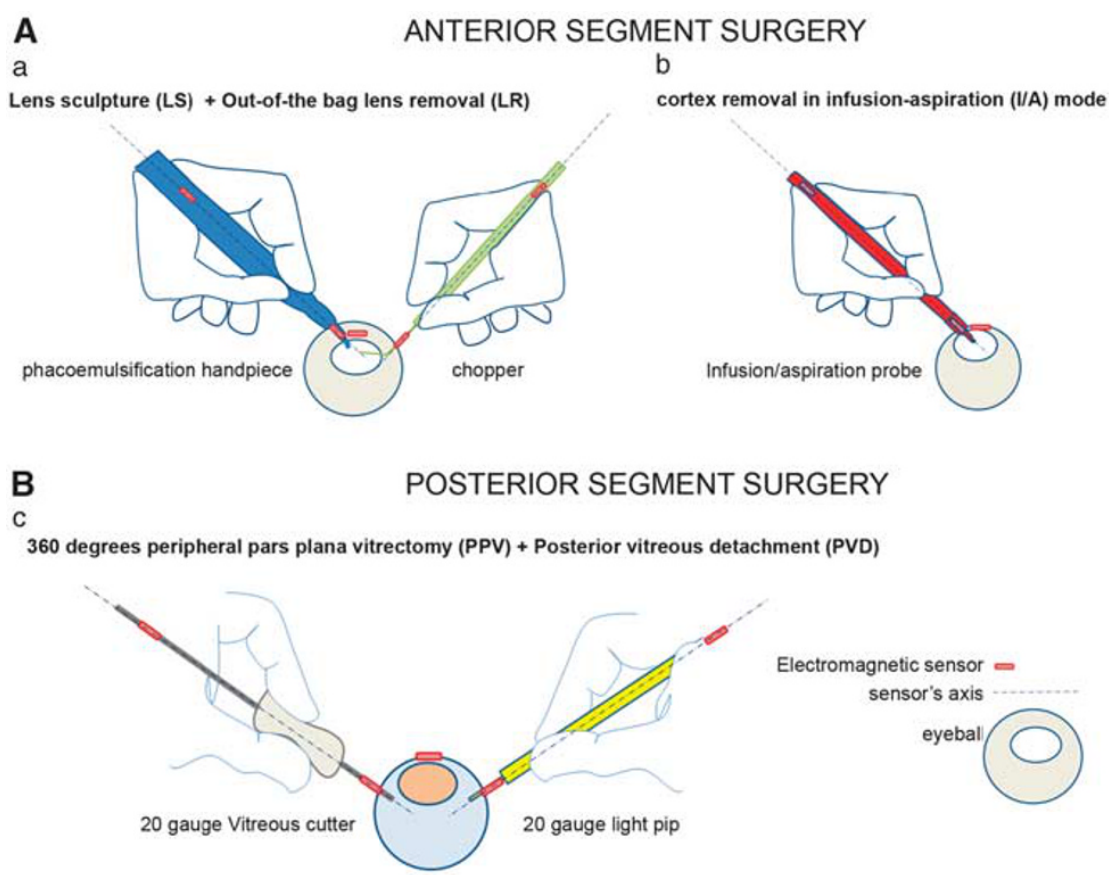

Figure 2 Experimental tasks tested with the sensors. The surgical steps of anterior segment surgery (a) and posterior segment surgery (b) are sketched. For the anterior segment surgery, the steps consisted of a LS and out-of-the bag LR performed with a phacoemulsification handpiece and a chopper $(\mathrm{A}, \mathrm{a})$, and a cortex removal in I/A performed with an I/A probe (A, b). The posterior segment steps consisted of a $360^{\circ}$ peripheral PPV and PVD both performed with a 20-gauge vitreous cutter and a 20-gauge light pipe and a vitreous cutter $(B, c)$. Each surgical instrument was equipped with a pair of electromagnetic sensors (red rectangles) and a referral sensor was positioned at the limbus of the eyeball being operated on. 


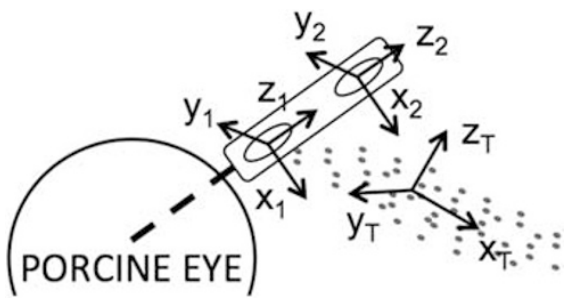

Figure 3 Electromagnetic sensors 1 and 2 are attached to the intraocular surgical tool shaft. Each sensor has its own internal $x$, $y$, and $z$ that can obtain information about the movement of the surgical tool as represented by gray dots (placed away from the tool to illustrate point). The raw data obtained in reference to $x 1$, $y 1, z 2, x 2, y 2$, and $z 2$ are transformed to $x \mathrm{~T}, y \mathrm{~T}$, and $z \mathrm{~T}$ using PCA. $x \mathrm{~T}$ is the axis with the greatest variance of the surgical tool, and $y \mathrm{~T}$ and $z \mathrm{~T}$ are orthogonal axes.

of the posterior hyaloid with the vitreous cutter to create a posterior vitreous detachment (PVD).

Five microsurgical instruments, namely a chopper (Katena Products, Denville, NJ, USA), a phacoemulsification handpiece plugged on an appropriate console (Millenium, Bausch and Lomb, San Dimas, CA, USA), an I/A probe (Bausch and Lomb), a 20-gauge Vitreous cutter, and a 20-gauge light pipe (Bausch and Lomb) were selected to perform the above procedures. To spatially track microsurgical instrument motion for translation and rotation, two seamless DC electromagnetic motion sensors (microBird) were stitched on the handle part of each intraocular surgical instrument, 2.5- and 7.5-cm away from the tip. Another sensor was positioned at the limbus of the porcine eyeball and secured in place with sutures to quantify eyeball motion.

\section{Software to record motions and plotting method}

Values of $x, y, z$ (in $\mathrm{mm}$ ) and azimuth, roll, yaw (in degrees) were recorded and saved in a database for subsequent range of motion analysis. As the sensors were attached on the tool 2.5 - and $7.5-\mathrm{cm}$ away from the instrument's tip to prevent compromise of surgical procedure, the offset from the tool tip to the sensor was found using a standard formula. ${ }^{6}$

Offset was calculated for every surgical tool with the assumption that the intraocular surgical tools are rigid bodies, which is reasonable given the gauge of the probe, which prevented flexion of the tools.

The $x, y, z$ axes of the electromagnetic transmitter do not best reflect the dynamics of the intraocular surgical motions. Therefore, a mathematical procedure called principal component analysis (PCA) was utilized. PCA is a mathematical procedure in which we take the gathered data and use orthogonal transformation to find the principal components. To analyze the motion of the tool
Table 1 Reliability of electromagnetic sensors for short and large translations in the three axes

\begin{tabular}{llllllll}
\hline \multirow{2}{*}{ Axis } & Length Mean & SD & \multicolumn{3}{c}{$95 \%$ LoA } & \multirow{2}{*}{ ICC } \\
\cline { 3 - 7 } & & & \multicolumn{2}{c}{ Left } & Right & In time & Across operators \\
\hline$x$ & Short & 5.978 & 0.135 & -5.713 & 6.244 & 0.365 & 0.545 \\
$x$ & Large & 0.308 & 0.035 & -0.240 & 0.376 & 0.194 & 0.767 \\
$y$ & Short & 8.405 & 0.112 & -8.185 & 8.625 & 0.119 & 0.796 \\
$y$ & Large & 3.052 & 0.040 & -2.973 & 3.130 & 0.933 & 0.999 \\
$z$ & Short & 8.058 & 0.151 & -7.761 & 8.354 & 0.967 & 0.668 \\
$z$ & Large & 0.281 & 0.065 & -0.153 & 0.409 & 0.970 & 1.000 \\
\hline
\end{tabular}

Abbreviations: 95\% LoA, 95\% limit of agreement; ICC, intraclass correlations; $\mathrm{SD}$, standard deviation.

shown in Figure 3, the sensors are placed on the shaft of the tool as stated above and will gather raw data of motion of the tool according to their internal axes $x 1, y 1$, $z 1, x 2, y 2$, and $z 2$. With PCA, the raw motion data of the tool, the vector from sensor 1 to sensor 2 (represented as dots in the figure for simplicity), is transformed to find the axis of the tool according to its maximum motion or high variance, $x \mathrm{~T}$, the principal component, and the subsequent orthogonal axes $y \mathrm{~T}$ and $z \mathrm{~T}$. Therefore, by using PCA, the internal structure of the surgical tool is revealed, instead of the tool motion data perceived by the internal axes of the sensor.

As the principal component obtained has the greatest variance, the maximum angles of tool were calculated; this became the true $x$ axis of the intraocular surgical tools. The next component with the greatest variance became the true $y$ axis, and the component with the least variance became the true $z$ axis. PCA was also performed with the referential sensor, with the principal component being the $x$ axis. The newly obtained data points showed movements along a curve, as the referential sensor was placed on the pig eye. The motion of the eye and the tools were analyzed separately to calculate the maximum range of motion.

Similar method was used to obtain the translational motion of the fulcrum point (entry site of instruments into the eye), which is located on the surface of the porcine eye.

Results were plotted using a tri-dimensional matrix either with the eye's fulcrum coordinates or with space coordinates as a referential.

\section{Statistics}

For reliability tests, descriptive statistics and intraclass correlations (ICC) were obtained from Excel software (Microsoft, Inc., Bellevue, WA, USA). Differences in motion among various surgical procedures or the subsets of surgical procedures were compared using Kruskal-Wallis 
Table 2 Rotation values in the three axes $(x, y, z)$ and translation values in the $z$-axis surgical of surgical instruments during various steps of intraocular surgery

\begin{tabular}{|c|c|c|c|c|c|c|c|}
\hline \multirow[t]{2}{*}{ Surgical step } & \multirow[t]{2}{*}{ Probe } & \multicolumn{2}{|c|}{$\mathrm{x}$-axis rotation (degrees) } & \multicolumn{2}{|c|}{$\mathrm{y}$-axis rotation (degrees) } & \multicolumn{2}{|c|}{$\mathrm{z}$-axis translation $(\mathrm{mm})$} \\
\hline & & $A v g \pm S D$ & Min-Max & $A v g \pm S D$ & Min-Max & $A v g \pm S D$ & Min-Max \\
\hline LS & Handpiece & $56 \pm 20$ & $38-98$ & $32 \pm 7$ & $18-38$ & $9 \pm 2$ & $7-12$ \\
\hline LS & Chopper & $23 \pm 11$ & $10-43$ & $46 \pm 14$ & $35-74$ & $16 \pm 13$ & $7-43$ \\
\hline LR & Handpiece & $60 \pm 24$ & 30-92 & $33 \pm 7$ & $23-46$ & $20 \pm 16$ & $8-53$ \\
\hline LR & Chopper & $21 \pm 19$ & $6-59$ & $31 \pm 29$ & $7-90$ & $10 \pm 9$ & $2-30$ \\
\hline $\mathrm{I} / \mathrm{A}$ & Probe & $72 \pm 14$ & $52-97$ & $48 \pm 10$ & $29-61$ & $15 \pm 5$ & $7-21$ \\
\hline PPV & Cutter & $96 \pm 44$ & $47-180$ & $142 \pm 39$ & $84-180$ & $30 \pm 5$ & $23-39$ \\
\hline PPV & Light & $38 \pm 8$ & $23-48$ & $64 \pm 10$ & $49-77$ & $19 \pm 4$ & $12-23$ \\
\hline PVD & Cutter & $81 \pm 26$ & 40-115 & $147 \pm 48$ & 61-180 & $24 \pm 6$ & $13-29$ \\
\hline PVD & Light & $23 \pm 5$ & $14-28$ & $25 \pm 6$ & $14-31$ & $11 \pm 4$ & $8-16$ \\
\hline
\end{tabular}

Abbreviations: Avg, average; I/A, infusion-aspiration; LR, lens removal; LS, lens sculpture; PPV, peripheral posterior vitrectomy; PVD, posterior vitreous detachment; SD, standard deviation.

Table 3 Calculated mean and maximal area of motion at the fulcrum (eye's entry site) during each task

\begin{tabular}{|c|c|c|c|c|c|c|c|c|c|c|}
\hline \multirow[t]{2}{*}{ Axis } & \multicolumn{2}{|c|}{$L S$} & \multicolumn{2}{|c|}{$L R$} & \multicolumn{2}{|c|}{$I A$} & \multicolumn{2}{|c|}{$P P V$} & \multicolumn{2}{|c|}{$P V D$} \\
\hline & $A v g \pm S D$ & Max & $A v g \pm S D$ & $\operatorname{Max}$ & $A v g \pm S D$ & $\operatorname{Max}$ & $A v g \pm S D$ & $\operatorname{Max}$ & $A v g \pm S D$ & Max \\
\hline$X\left(\mathrm{~mm}^{2}\right)$ & $6.27 \pm 2.33$ & 9.79 & $5.56 \pm 2.22$ & 9.22 & $12.15 \pm 4.3$ & 12.58 & $6.57 \pm 3.66$ & 11.85 & $6.14 \pm 3.03$ & 11.09 \\
\hline $\mathrm{Y}\left(\mathrm{mm}^{2}\right)$ & $3.56 \pm 1.74$ & 6.30 & $3.68 \pm 1.46$ & 5.88 & $6.93 \pm 2.18$ & 8.87 & $5.85 \pm 2.84$ & 7.84 & $3.29 \pm 1.13$ & 6.03 \\
\hline $\mathrm{Z}\left(\mathrm{mm}^{2}\right)$ & $1.74 \pm 0.55$ & 2.50 & $1.46 \pm 0.31$ & 1.97 & $2.18 \pm 1.10$ & 4.12 & $2.84 \pm 1.63$ & 4.13 & $1.13 \pm 0.61$ & 1.99 \\
\hline
\end{tabular}

Abbreviations: Avg, average; I/A, infusion-aspiration; LR, lens removal; LS, lens sculpture; Max, maximal value; PPV, peripheral posterior vitrectomy; $\mathrm{PVD}$, posterior vitreous detachment; SD, standard deviation.

tests. Statistical analysis was performed using SAS software version 9.1 (SAS, Inc., Cary, NC, USA).

\section{Results}

Reliability of the electromagnetic motion capture device

Reliability results for electromagnetic sensors are shown in Table 1. For translations, the reproducibility across operators (between operator 1 and 2) was good to near perfect for both short and large translations and across the three axes (mean ICC $=0.796$, from 0.545 to 1 ).

\section{Translation and rotation of surgical tools during phacoemulsification standard steps}

Figure 2 displays the position and maximal rotation of each instrument in the $x$ and $y$ axes around the average position and maximal translations in the $z$ axis for each surgical step of predefined intraocular surgery.

Translations and rotations are provided in Table 2 with respect to instruments and surgical steps. In the $z$ axis, the overall maximal translation was $53 \mathrm{~mm}$. It was obtained with the phacoemulsification handpiece during lens nucleus removal. The overall minimal translation was $2 \mathrm{~mm}$ in this axis, obtained with the chopper during the LR step of phacoemulsification. Maximal rotations in the $x$ and $y$ axes were obtained during PPV and PVD with the vitreous cutter, respectively $96 \pm 44^{\circ}, 142 \pm 39^{\circ}$ in the $x$ axis; $81 \pm 2^{\circ}$ and $147 \pm 48^{\circ}$ in the $y$ axis. Minimal translations and rotations were recorded with the chopper during anterior segment surgery, and the light probe during posterior segment surgery.

Both average and maximal rotations (in the $x$ and $y$ axes) and translations (in the $z$ axis) were statistically higher with the dominant operating hand (Phacoemulsification handpiece, vitreous cutter) when compared with the second hand (chopper, light pipe) (Kruskal-Wallis test $P$-value: $P<0.0001$ in the $x$ axis, $P=0.004$ for in the $y$ axis, $P=0.005$ in the $z$ axis).

We observed that the dominant hand's rotations in the $y$ axis and translations in the $z$ axis during posterior surgical steps exceeded the ones from anterior surgical steps $(P=0.12$ in the $x$ axis, $P<0.0001$ in the $y$ axis, $P=0.007$ in the $z$ axis). Differences obtained with the second hand did not reach statistical significance in the three $x, y$, and $z$ axes $(P=0.11, P=0.16$, and $P=0.061$, respectively).

During all performed tasks, the fulcrum moved within a $12.58 \mathrm{~mm}^{2}$ maximal area in the $x, y$, and $z$ axes (Table 3). The translation of the main hand was statistically superior in the three axes $(x, y$, and $z)$ than the second hand in the two posterior surgeries tasks tested, 


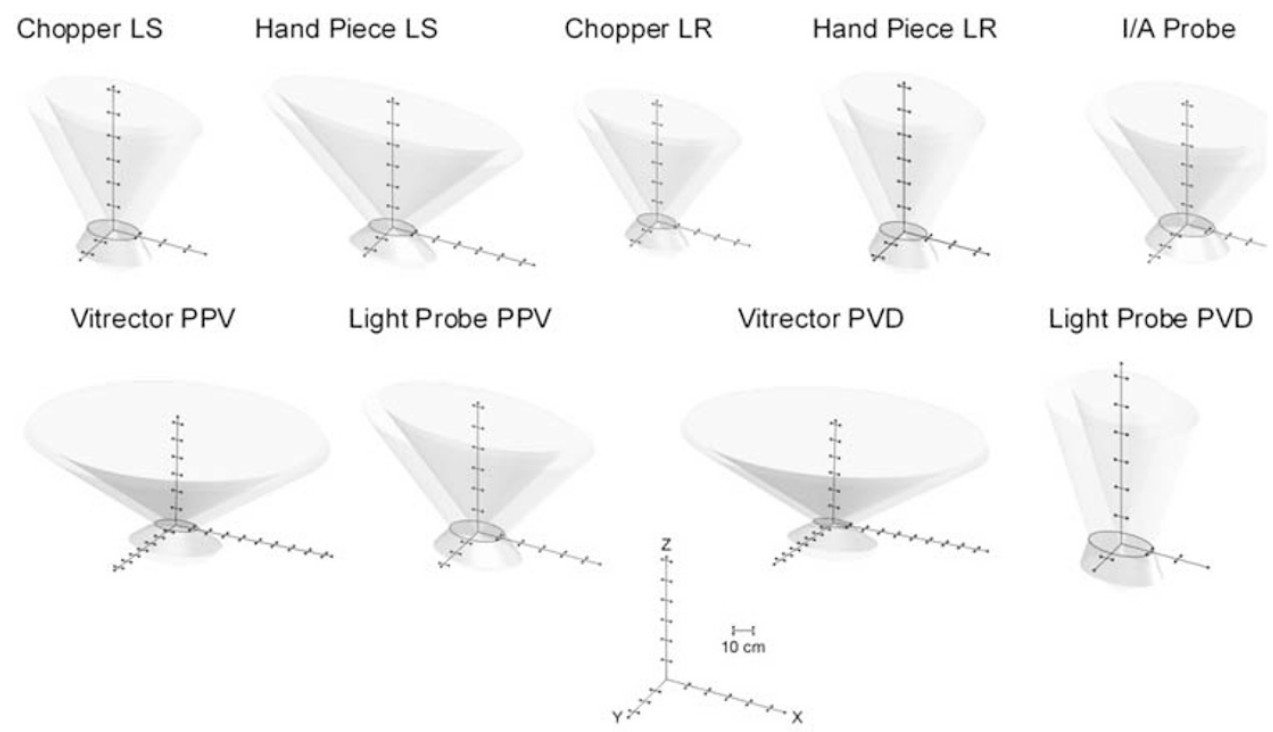

Figure 4 Each plot displays the average rotation and translation observed a single surgical instrument during a specified surgical step of surgical procedure. Rotations are displayed through three-dimensional ellipses. Ellipse axes are centered on average angulations, which are arbitrarily materialized with the $z$ axis. Edges of ellipses display maximal rotation observed during the whole surgical step. When the entry site location (coordinate zero) is taken for referential point for calculations, ellipses are colored in yellow and figure instrument's angulations from a surgeon's point of view (into the eye). When raw space coordinates are used for calculations, ellipses are colored in a translucid gray figure instrument's angulations for robot consideration (mechanical clearance). There, the gray oval line drawn on the $x$ and $y$ axes and centered on coordinate zero figures the eye motion itself. The color reproduction of this figure is available on the html full text version of the manuscript.

corresponding to PPV $(P=0.007$ for $x, P=0.004$ for $y$, $P=0.007$ for $z)$ and $\operatorname{PVD}(P=0.004$ for $x, P=0.004$ for $y$, $P=0.004$ for $r, P=0.010$ for $z$ ). As illustrated in Figure 4, the translation of the entry site did not differ significantly for any of the axis, whether operators performed anterior or posterior segment surgery $(P=0.90$ for $x, P=0.97$ for $y$, $P=0.58$ for $z$ ).

\section{Discussion}

Robotic surgery belongs to microinvasive surgical techniques, and is now widely accepted as a valuable alternative to conventional surgery in many surgical fields such as urology, cardiology, and digestive surgery. However, robotic systems are facing challenges in ophthalmology and are, thus, not currently available for intraocular surgery. To perform intraocular surgery, conflicting needs have to be handled simultaneously. Even though a wide range of motion is required, the intraocular instruments are constrained at a single point located at the eye's entry site. Usually, two entry sites are mandatory to perform bimanual intraocular surgery. These entry sites, called fulcrum, are necessarily close to each other because of anatomical considerations. These basic specificities should be integrated to the design of the remote center of motion (location in space where the surgical instrument is constrained to pass through) of any robotic platform dedicated for intraocular surgery.
Various robotic systems have been designed to assist ocular surgeons, but they are mainly dedicated to a single surgical task. So far, systems have been designed to primarily emphasize degrees of freedom (DoF) allowed by robotic arms, along with the idea that the optimal system would allow for maximal range of motion and DoF. As a drawback, large DoF subsequently implies that the system lacks in precision, reliability, and is subjected to potential hazards when unnecessary or unrewarded motion is permitted.

An objective, quantified, and accurate evaluation of the range of motion during intraocular surgery is another approach to initiate the design of a new tailored eye robotic surgical platform. This assessment could also better define the capabilities of existing robotic surgical systems, as well as the tasks they might be able to achieve. In this evaluation, data are provided in the three axes of space $(x, y$, and $z)$ with the range of motion of each instrument, as well as the fulcrum during the five main surgical steps of intraocular surgery (LS, LR, irrigation aspiration for cataract extraction and PVD, peripheral vitrectomy for vitreoretinal surgery) to help engineers design a new robotic microsurgical platform.

Rotation and translation, when performed by a robot, correspond to different requirements. The robot's bulkiness depends mainly on rotations. Our data shows how the requirements for rotations could be different 
depending on the surgical steps of a single procedure. For instance, during phakoemulsification, the LS would need a motion allowance with higher angle of rotation than LR. In addition, rotation angles are markedly different whether the considered surgical instrument achieves a main or an ancillary task. This is particularly noticeable for the vitreous cutter on one side and the light probe on the other during vitrectomy (Figure 4).

Besides electromagnetic sensors, the other types of sensors such as optical or inertial sensors (accelerometers, magnetometers, and gyroscopes) might have also been considered, as they confer an equivalent level of accuracy. ${ }^{7}$ However, electromagnetic sensors were chosen in our experiment because of their ability to provide data in burdened areas, as well as their small size, which made their embedment not interfere with intraocular surgical procedures. Optical sensors require a line of sight difficult to guarantee during intraocular surgery, given the limited space of maneuver. Inertial sensors are usually bulky, and modify the gripping ability of the instruments and precision of microsurgical gestures. The major drawback of electromagnetic sensors resides in the magnetic field distortion because of metallic instruments nearby, but was addressed by the manufacturer (Ascension) through the use of specificpulsed DC signals in the active source. ${ }^{8}$ However, to confirm the accuracy and reproducibility of our electromagnetic sensors in our laboratory, an evaluation was performed and it demonstrated a near-to-perfect reproducibility across operator. Reproducibility in time was not as good as across operator, probably because of magnetizable objects variably placed in the vicinity of the electromagnetic source. We, therefore, conducted experiments on porcine eye within a single session at a steady-state environment, particularly focusing on the proximity of magnetizable objects to both sensors and the electromagnetic source.

Five representative surgical tasks of lens extraction and vitrectomy surgery were performed to assess the range of motion of the instruments and eyeball in the $x, y$, and $z$ axes. We observed a wide variability in the range of motion (rotation and translation) depending on the hand or the surgical task studied. To define a framework of specifications for ocular robotic surgical platform, figuring out what range of motion the surgical instruments and the eyeball are subject to during intraocular surgical procedures is critical. Interaction and placement of each instrument toward each other are also critical data. On the other hand, a robotic surgical assistance should be able to perform the same task, regardless the operating arm used. We should, therefore, consider a surgical platform that allows the maximal range of motion measured for each robotically guided instrument.
In addition, the subsequent analysis of such data may also help for ophthalmologic surgical evaluation and training. Quantifications of surgical motions might provide reproducible assessments for surgical skills. Such an objective tool could record operating motion from a single surgeon to compare both of his hands with regard to specified surgical steps. Novice surgeons could also be evaluated or compared with each other or with trained surgeons.

Intraocular surgery requires bimanual surgical abilities. However, the dominant hand offers, for non-ambidextrous people, a better control, and in most cases, the surgeon will prefer to perform the most challenging part of the surgery with this hand and will mostly use the second hand as assistance. Our results showed minimal translations and rotations for the second hand (chopper for anterior segment surgery and light probe for posterior segment surgery), and demonstrated that maximal rotation ( $x$ and $y$ axes) and translation ( $z$ axis) were significantly higher with the dominant hand when compared with the second hand in every surgical step. The different uses of both hands were particularly evident during vitrectomy because the light pipe is usually kept in the central part of the vitreous cavity compared with the tip of the vitreous cutter, which has to move along the retinal surface. This was confirmed by statistical analysis in which translations of the dominant hand across the three axes $(x, y$, and $z$ ) were higher than those for the second hand (light pipe) during all vitrectomy tasks.

Cataract surgery and vitrectomy are performed in two separate zones of different volumes. The bigger size of the vitreous cavity compared with the anterior segment explains the wider motion of the intraocular instrument to achieve the surgical task. It is, thus, not surprising that our measurements demonstrated maximal rotations in the $x$ and $y$ axes during PPV and PVD obtained with the vitreous cutter. The same volume consideration would further explain the more pronounced rotation ( $y$ axis) and translation ( $z$ axis), with the dominant hand during vitrectomy, when compared with cataract surgery. However, the lack of any statistical difference with the second hand (chopper vs light pipe) illustrates how, regardless of the surgical task performed, the secondhand motions will always be limited.

During intraocular surgery, instruments are introduced through the cornea or the sclera to reach the desired intraocular surgical field. Fulcrum number, location, and motions during intraocular surgery should carefully be defined to design an ocular robotic platform. Slight rotation and translation of the eyeball can help to improve visibility or access to the surgical field during intraocular procedures. However, excessive lateral stretch applied at the entry site may result in tissue 
damages. We measured low and symmetrical translation values at the fulcrum during the different surgical tasks.

In conclusion, the quantitative data provided by this evaluation helps to design new robotic systems for surgical assistance in ophthalmology as well as to better define what existing ocular surgical systems are capable of. The data collected in this study could also be of interest to develop teaching or training tools for surgeons during ocular procedures.

\section{Summary}

What was known before

- Rough evaluation of the surgical motion of the instruments during intraocular surgery.

\section{What this study adds}

- Accurate evaluation of the motion of the surgical instruments during intraocular surgery.

- Comparison of the range of motion needed for the main hand $v$ s the ancillary one.

- Comparison of the range of motion needed for anterior segment surgery vs vitreo-retinal surgery.

- Give specifications for the design of a new surgical ocular robotic platform.

\section{Conflict of interest}

The authors declare no conflict of interest.

\section{Acknowledgements}

We thank Fei Yu, PhD, for his assistance with statistical analysis.

\section{References}

1 Tsirbas A, Mango C, Dutson E. Robotic ocular surgery. Br J Ophthalmol 2007; 91: 18-21.

2 Bourges J-L, Hubschman J-P, Burt B, Culjat M, Schwartz S. Robotic microsurgery: corneal transplantation. $\mathrm{Br} J$ Ophthalmol 2009; 93(12): 1672-1675.

3 Bourla DH, Hubschman JP, Culjat M, Tsirbas A, Gupta A, Schwartz SD. Feasibility study of intraocular robotic surgery with the da Vinci surgical system. Retina 2008; 28: 154-158.

4 Hubschman J, Bourla D, Tsirbas A, Culjat M, Dutson E, Kreiger AE, Schwartz SD. Robotic vitreoretinal surgery. Sci IOV. ARVO Annual Meeting. Fort Lauderdale. Invest Ophthalmol Vis Sci 2007; 48: E-Abstract pp 6030.

5 Yu DY, Cringle SJ, Constable IJ. Robotic ocular ultramicrosurgery. Aust N Z J Ophthalmol 1998; 26(Suppl 1): S6-S8.

6 Goldstein H, Poole CP, Safko JL. Classical Mechanics, 3rd ed. Addison Wesley: San Francisco, 2002. ISBN 978-0-201-65702-9.

7 Saber-Sheikh K, Bryant E, Glazzard C, Hamel A, Lee R. Feasibility of using inertial sensors to assess human movement. Man Ther 2010; 15: 122-125.

8 Foxlin E. Motion tracking requirements and technologies. In: Associates LE (ed.). Handbook of Virtual Environment Technology. K Stanney: New York, 2002, pp 163-210. 